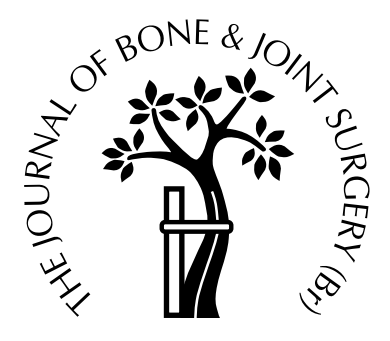

\title{
Dupuytren's disease and frozen shoulder induced by treatment with a matrix metalloproteinase inhibitor
}

\author{
J. W. Hutchinson, G. M. Tierney, S. L. Parsons, T. R. C. Davis \\ From Queen's Medical Centre, Nottingham, England
}

I n a series of 12 patients with inoperable gastric carcinoma who had treatment with a synthetic matrix metalloproteinase inhibitor (Marimastat) for more than one month, six developed a frozen shoulder or a condition resembling Dupuytren's disease.

This suggests that the matrix metalloproteinases, a family of naturally occurring proteinases, may be involved in the pathogenesis of these two conditions. Our observation opens avenues for further research which could lead to local or systemic therapeutic interventions for frozen shoulder and Dupuytren's disease.

J Bone Joint Surg [Br] 1998;80-B:907-8.

Received 16 October 1997; Accepted after revision 3 April 1998

The pathogenesis of both Dupuytren's disease and frozen shoulder is poorly understood, but they have very similar histopathological and immunocytochemical features ${ }^{1}$ and associations with other conditions such as hyperlipidaemia ${ }^{2}$ and diabetes. ${ }^{3}$

The matrix metalloproteinases (MMPs) are zincdependent proteinases which in normal tissues degrade the connective-tissue matrix as part of normal turnover. In gastric carcinoma there is an overexpression of MMPs which leads to an increase in the breakdown of connective tissue and a possible enhancement of tumour invasion.

In a recent phase-II study of the effect of the synthetic broad-spectrum MMP inhibitor, Marimastat (British Biotech Ltd, Oxford, UK), in a dosage of 25 to $100 \mathrm{mg}$ per day, on patients with advanced gastric cancer, 4 it was found that a number developed a frozen shoulder and a hand condition resembling Dupuytren's disease. This suggests

J. W. Hutchinson, FRCS, Specialist Registrar in Orthopaedics

G. M. Tierney, FRCS, Specialist Registrar in General Surgery

S. L. Parsons, FRCS, Specialist Registrar in General Surgery

T. R. C. Davis, FRCS, Consultant Orthopaedic Surgeon

University Hospital, Queen's Medical Centre, Nottingham NG7 2UH,

UK.

Correspondence should be sent to Mr J. W. Hutchinson at 23 Charnock Avenue, Wollaton Park, Nottingham NG8 1AG, UK.

(C)1998 British Editorial Society of Bone and Joint Surgery $0301-620 \mathrm{X} / 98 / 58464 \$ 2.00$

VOL. 80-B, No. 5, SEPTEMBER 1998 that alterations in MMP activity may be involved in the pathogenesis of these two conditions.

\section{Case reports}

Between June 1995 and September 1996 we recruited 24 patients with inoperable, histologically proven primary or recurrent gastric adenocarcinoma into the study. The trial protocol was approved by the Research Ethics Committee. All patients provided witnessed, written informed consent to participate in the study which was conducted in accordance with the European Guidelines on Good Clinical Practice.

Before beginning treatment all patients had routine serum blood tests and a full physical examination. They were reassessed monthly in regard to the response to treatment and the presence of adverse conditions. Once a pattern of shoulder and hand conditions had emerged, we then assessed the musculoskeletal system in all patients both before beginning treatment and at the monthly review.

Of the 24 patients, 12 were judged to have had a favourable response to treatment with Marimastat and continued to receive it beyond the initial one-month assessment period. After four months of treatment three of these 12 patients developed clinical features of Dupuytren's disease of the hand and frozen shoulder, as defined by Codman in $1934,{ }^{5}$ and another three developed a frozen shoulder alone. In all cases the frozen shoulder was bilateral. Of the three patients with the Dupuytren-type condition two developed contractures with palmar cords and pits involving the little, ring and middle fingers (Fig. 1), and the other palmar nodules only. Unfortunately, no autopsies were performed and therefore no tissue was available for analysis.

The acute symptoms (pain and stiffness) of frozen shoulder improved when treatment with Marimastat was temporarily discontinued, but became worse again when it was recommenced.

\section{Discussion}

MMPs are a family of zinc-dependent proteinases the primary function of which is to degrade the extracellular matrix. They are found in normal tissues and regulate the turnover of the connective tissue matrix. ${ }^{6}$ The synthesis and 


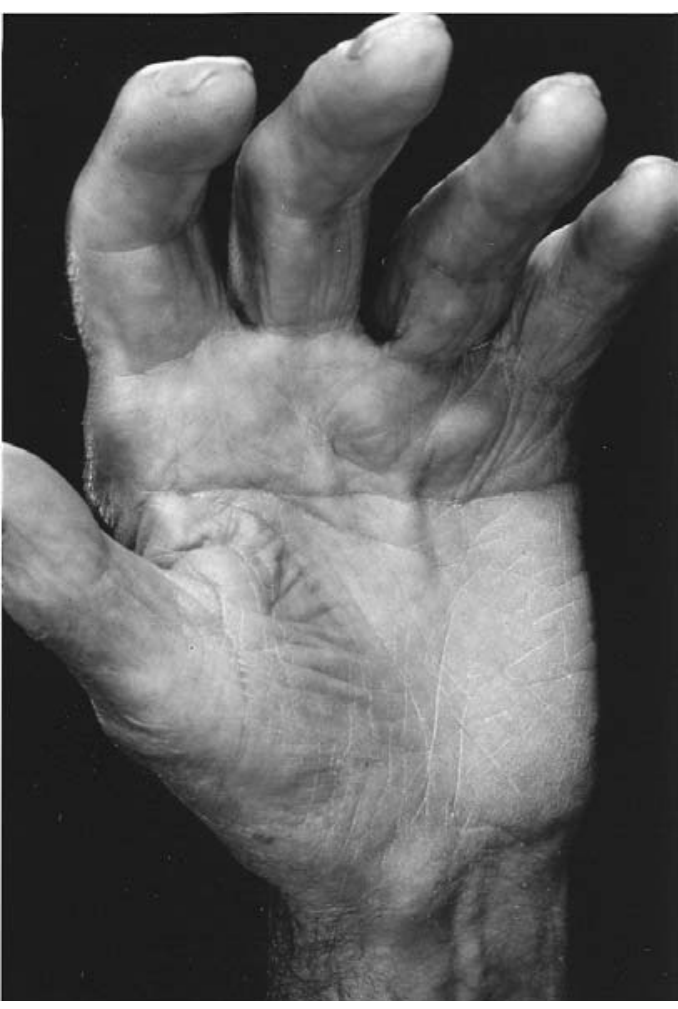

Fig. 1

The hand of a 71-year-old man seven months after beginning treatment with Marimastat showing the cords, pits and contractures.

activity of MMPs are controlled by specific tissue MMP inhibitors (TIMPs) and a variety of cytokines and growth factors.

The MMP:TIMP ratio has been shown to be important in various pathological processes such as invasion by gastric and colorectal tumours ${ }^{7}$ and is increased in rheumatoid arthritis. ${ }^{8}$ MMPs have been implicated in the breakdown of the basement membrane which may facilitate tumour invasion and entry into blood vessels and lymphatics. ${ }^{7}$ Primary cell cultures of pig fibroblasts from radiation-induced dermal fibrosis show reduced MMP-1 and increased TIMP expression and accumulate large amounts of collagen, proteoglycans and fibronectin. ${ }^{9}$ MMP synthesis is tightly regulated by a number of cytokines and growth factors such as transforming growth factor-beta, tumour necrosis factor, interleukin-1 and oncostatin $\mathrm{M} .^{10}$ Abnormal levels of these factors have been observed in Dupuytren's tissue. ${ }^{11}$
It is postulated that the development of frozen shoulder and the Dupuytren-type condition in our patients was due to a decrease in the MMP:TIMP ratio which caused increased synthesis and deposition of collagen and connective tissue. A decrease in the MMP:TIMP ratio may therefore represent a common pathway in the pathogenesis of Dupuytren's disease and frozen shoulder. In normal circumstances this ratio may be controlled by a variety of cytokines, growth factors and genetic factors. Although no histopathological analysis was possible and it is not proven that Marimastat caused these hand and shoulder changes, our observation suggests possible therapeutic strategies for these common conditions involving manipulation, either locally or systemically, of the MMP:TIMP activity.

The gastric cancer study was sponsored by British Biotech Pharmaceuticals Ltd, Oxford, UK.

Although none of the authors have received or will receive benefits for personal or professional use from a commercial party related directly or indirectly to the subject of this article, benefits have been or will be received but are directed solely to a research fund, educational institution, or other non-profit institution with which one or more of the authors is associated

\section{References}

1. Bunker TD, Anthony PP. The pathology of frozen shoulder syndrome: a Dupuytren-like disease. J Bone Joint Surg [Br] 1995;77-B: 677-83.

2. Bunker TD, Esler CN. Frozen shoulder and lipids. J Bone Joint Surg [Br] 1995;77-B:684-6.

3. Noble J, Heathcote JG, Cohen H. Diabetes mellitus in the aetiology of Dupuytren's disease. J Bone Joint Surg [Br] 1984;66-B:322-5.

4. Parsons SL, Watson SA, Griffin NR, Tierney GM, Steele RJC. A phase I/II study of the oral matrix metalloproteinase inhibitor, Marimastat, in patients with inoperable gastric cancer. Gastroenterology 1997;112:636.

5. Codman EA. Tendonitis of the short rotators. In: Ruptures of the supraspinatus tendon and other lesions in or about the subacromial bursa. Boston: Thomas Todd and Co, 1934.

6. Murphy G. Matrix metalloproteinases and their inhibitors. Acta Orthop Scand Suppl 1995;266:55-60.

7. Parsons SL, Watson SA, Brown PD, Collins HM, Steele RJ. Matrix metalloproteinases. Br J Surg 1997;84:160-6.

8. Hembry RM, Bagga MR, Reynolds JJ, Hamblen DL. Immunolocalisation studies on six matrix metalloproteinases and their inhibitors, TIMP-1 and TIMP-2, in synovia from patients with osteo- and rheumatoid arthritis. Ann Rheum Dis 1995;54:25-32.

9. Lafuma C, El Nabout RA, Crechet F, Hovnanian A, Martin M. Expression of 72-kDa gelatinase (MMP-2), collagenase (MMP-1), and tissue metalloproteinase inhibitor (TIMP) in primary pig skin fibroblast cultures derived from radiation-induced skin fibrosis. J Invest Dermatol 1994;102:945-50.

10. Richards CD, Shoyab M, Brown TJ, Gauldie J. Selective regulation of metalloproteinase inhibitor (TIMP-1) by oncostatin M in fibroblasts in culture. J Immunol 1993;150:5596-603.

11. Baird KS, Crossan JF, Ralston SH. Abnormal growth factor and cytokine expression in Dupuytren's contracture. J Clin Pathol 1993; 46:425-8. 